\title{
A Review of Excitation-Inhibition Balance in the Nucleus Tractus Solitarius as a Gateway to Neural Cardiovascular Regulation
}

\author{
Masoumeh Kourosh-Arami $^{*(i D)}$, Monireh Mohsenzadagan ${ }^{2}$ (D), Alireza Komaki ${ }^{3}$ (D)
}

1. Dept. of Neuroscience, School of Advanced Technologies in Medicine, Iran University of Medical Sciences, Tehran, Iran

2. Dept. of Laboratory Sciences, School of Allied Medicine, Iran University of Medical Sciences, Tehran, Iran

3. Neurophysiology Research Center, Hamadan University of Medical Sciences, Hamadan, Iran

\begin{tabular}{|c|}
\hline Article Info \\
\hline doi $10.30699 / j a m b s .28 .126 .47$ \\
\hline $\begin{array}{l}\text { Received: 2019/10/20; } \\
\text { Accepted: 2019/12/28; } \\
\text { Published Online: 05 Jan 2020; }\end{array}$ \\
\hline $\begin{array}{l}\text { Use your device to scan and read the } \\
\text { article online }\end{array}$ \\
\hline 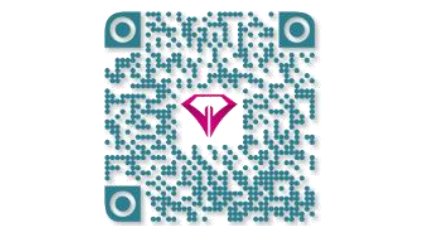 \\
\hline
\end{tabular}

Corresponding Information:

Masoumeh Kourosh-Arami,

Dept. of Neuroscience, School of Advanced

Technologies in Medicine, Iran University of

Medical Sciences, Tehran, Iran

E-Mail: kourosharami.m@iums.ac.ir

\begin{abstract}
Physiological experiments show that mean blood pressure is controlled by the nervous system in long-term. The nucleus tractus solitarius (NTS), located in the dorsomedial medulla oblongata is extensively recognized as an essential brain area complicated in the integration of numerous viscerosensory processes, such as respiratory, cardiovascular, hepatic gustatory, and renal regulation mechanisms. NTS is a region of the brain stem in which primary baroreceptor afferents terminate and synapse with the rostral ventrolateral medulla (RVLM) via a nitric oxidergic pathway and hence is vital in the normal control of arterial pressure (AP). The NTS as a comparator evaluates the error signals between afferents of cardiovascular receptor and central neural structures and sends signals to nuclei that normalize the circulatory variables. Furthermore, during exercise, signals from the muscle receptors reach the NTS that activate sympathetic premotor neurons and thus cause pressor and tachycardiac responses. The GABAergic interneurons of NTS may contribute to baroreceptor reflex resetting by the inhibition of the barosensitive NTS neurons, thereby enhancing the sympathetic nerve activity. The basic functions of the NTS with respect to regulating the cardiovascular system are introduced in this review. Then, the potential mechanisms underlying cardiovascular regulation are discussed with a focus on NTS functions.
\end{abstract}

Keywords: Cardiovascular system, GABA, Glutamate, Nitric oxide, Nucleus tractus solitarius

(c) (1) (8) Copyright $(\odot 2020$, This is an original open-access article distributed under the terms of the Creative Commons Attribution-noncommercial 4.0 International License which permits copy and redistribution of the material just in noncommercial usages with proper citation.

\section{Introduction}

The NTS is a critical constituent for the homeostasis of autonomic function since it is innervated by visceral inputs from peripheral receptors of gastrointestinal, gustatory, cardiovascular, and pulmonary/respiratory systems, which reflexly influence autonomic motor outputs (1).

The NTS compares the signals between afferents of cardiovascular receptor and central neural structures and sends error signals to nuclei that normalize the circulatory variables (2).

The NTS reflexly regulates arterial pressure (AP) and heart rate (HR) to sustain cardiovascular homeostasis; moreover, this nucleus is involved in feed-forward control of the cardiovascular system in reaction to an array of mental and physical stressors (1).

The AP is adjusted by the feedback of chemoreceptors and cardiopulmonary receptors, and the baroreflex would make the short-term pressure constant. The set point of the open and closed loops of cardiovascular regulation is the firing rates of rostral neural afferents to the NTS. Thus, the RNS has an effective action not only inconstant conditions, but also in diverse behaviors and pathologies (2). The regulation of AP cannot be accomplished after chemo and cardiopulmonary receptor denervation (1).

Cardiovascular variables are regulated by humoral, neural, and autoregulatory mechanisms. It is generally accepted that the renal output is involved in the maintenance of the long-term AP, but the nervous system plays a possible role in this process.

Guyton reported that hypertension in human is mostly started by nervous stress (2). Evaluations of AP illustrate large deviations over a 24-hr period. It is reported that acute emotional or threatening stimuli can produce a noticeable cardiovascular response, as in the alert response (3). Electrical stimulation of defense region in 
the hypothalamus induces a cardiovascular response similar to that described above. In addition, the beginning of exercise is pursued and occasionally preceded by instant enhancements of AP, HR, and ventilation. The latter is accompanied by a rise in the activity of sympathetic nerves to vascular beds, such as the kidneys (4). The cardiovascular alterations that happen at the start of exercise result from central command of the cortex. Thus, the set point of AP varies in diverse conditions. Osborn et al. (5) found that a "baroreflex independent" sympathetic regulation should occur for the lasting control of sympathetic nerve activity and AP, conferring correspondingly the idea of a central nervous system "set point" and its participation in the pathogenesis of hypertension. Therefore, there is a neural set point for the long-term control of blood pressure $(2,5)$. In our previous research, it was found that NTS could decrease diastolic pressure in patients with diabetes by injection of lidocaine into NTS and thus reversible inactivation (6).

The NTS is situated at the dorsomedial medulla that receives general visceral and taste afferents and has an important effect on the initiation of reflexes directing cardiovascular function, respiration, and gastrointestinal motility (7). Furthermore, the NTS might be one of the important centers as a sensory gateway from the periphery to the central system that is complicated in both somatic and visceral motor systems (8). Cui et al. found that some interneurons in the NTS might have a predominantly significant role in the cardiomodulatory process. They showed that electroacupuncture may diminish myocardial ischemic injury by modifying the activity of the vagus nerves to NTS (9).

In this review, the basic functions of the NTS are introduced with respect to regulating the cardiovascular system. Then, the potential mechanisms underlying cardiovascular regulation are discussed during a single bout of exercise, with a focus on NTS functions.

GABAergic neurons are scattered in the NTS and their tonic activity is essential for the regulation of cardiorespiratory activities (10). Injection of GABAA and $\mathrm{GABAB}$ agonists, muscimol and baclofen respectively into the NTS increased AP. Furthermore, intra-NTS microinjection of bicuculline as a GABAA receptor antagonist diminished AP. GABA in the NTS has a significant effect on controlling AP, particularly over $\mathrm{GABAB}$ receptors, and shows an inhibitory role in the baroreceptor reflex (10). The GABAergic inhibition is regulated by the baroreceptors excitatory synaptic input to the NTS (11).
The NTS collects afferent protrusions from the cardiopulmonary receptors, carotid chemoreceptors, and arterial baroreceptors, and regulates the autonomic activity to keep AP in a constricted range of variation. NTS receives viscerosensory afferents that are glutamatergic (12). The stimulation of these cardiovascular afferents induces a precise autonomic response through the stimulation of neuronal efferents from the NTS to the caudal ventrolateral zone of the medulla (CVLM) and RVLM (13). Stimulation of the cardiovagal component through cardiovascular reflex or viaintra-NTS application of glutamate (GLU) is arbitrated to N-methyl-D-aspartate (NMDA), and the sympatho-excitatory constituent of the chemoreflex and the pressor response to GLU injected into the NTS cannot be altered through an NMDA receptor antagonist; this demonstrates that the sympathoexcitatory constituent of these responses is arbitrated to non-NMDA receptors (13). It is reported that the alteration of NMDA percentage may show some effects on pressor response to GLU, similar to their effect on plasticity (14).

Excitatory neurotransmitters, such as L-glutamate, are the core neurotransmitters in the regulation of AP by NTS (10). It is revealed that in the CNS, the GLU as the main excitatory neurotransmitter, acts on ionotropic receptors, AMPA and NMDA, with the latter having a higher affinity for L-glutamate than AMPA receptors $(\mathbf{1 5}, \mathbf{1 6})$. Likewise, GABA is the primary inhibitory neurotransmitter. Both GLU and GABA in the brainstem and spinal circuits show crucial roles in cardiovascular control by the CNS (17).

The level of inhibition by GABA in cardiorespiratory circuits of the NTS may be reliant on the action of the excitatory interneurons. Also, the ratio of excitatory to inhibitory action may regulate the spread of signals over the NTS neuronal network. This type of dependence of inhibitory GABAergic neurons on the activity of the surrounding excitatory neurons has been extensively shown in the cortex $(\mathbf{1 8 , 1 9 )}$ and termed 'activity dependence'(11).

The rate of GABAergic inhibition within the NTS increases in a high blood pressure $(20,21)$ and hence the resultant elevation of GABAergic inhibition within the NTS causes increases in HR, AP, and sympathetic outflow (22). These properties are parallel to the inhibitory action of the arterial baroreceptor reflex (23). Therefore, our findings indicated that disinhibition of the NTS circuit may lead to higher cardiovascular responses in the hypertensive rat. 
Sapru et al. demonstrated that injection of glutamate into the NTS reduced cardiovascular responses similar to baroreceptor reflexes. Baroreceptor afferent nerves from aortic arch and carotid sinus send excitatory signals to the NTS through $\mathrm{IX}^{\text {th }}$ and $\mathrm{X}^{\text {th }}$ cranial nerves. This action causes the inhibition of sympathetic preganglionic vasomotor neurons of the spinal cord (17).

Previous studies have shown that augmented excitability of GABAergic neurons in the NTS during temporal lobe epilepsy and hence NTS dysfunction may contribute to the increased probability for cardiorespiratory collapse in epilepsy $(24,25)$.

\section{Early and Late Neurons of NTS Control Blood Pressure Changes}

Previously, NTS barosensitive neurons were divided to early and late neurons according to their discharge rates, with the former displaying a greater discharge rate than the latter throughout AP increase at a similar rate. The firing rate of early neurons linearly increases with the initial HR diminution while that of late neurons increases with the late phase of HR reduction (26).

According to Rogers et al., NTS neurons not only are sensitive to AP, but also encode the rate of AP alteration (27). It was later reported by Zhang and Mifflin that different rates of AP alteration might cause different neuronal reactions in the same NTS neurons (28): a fast augmentation rate of AP may cause a greater response relative to a slow rate increment. A report by Deuchars et al. suggests that NTS barosensitive neurons are heterogeneous in physiological action and morphological form (29). Peak firing rate, the firing patterns, and time course of the response to AP changes are different between diverse NTS barosensitive neurons. The NTS neurons were detected with diverse patterns of firing: excitatory (mostly), inhibitory, or biphasic (minimally). The excitatory neurons are grouped to early and late neurons based on their response time course (30). The early neurons rapidly boost their discharge throughout the early phase of AP increment, whereas late neurons do not increase their discharge until near/after the peak of AP. Early neuron action in response to AP increase causes an expressively greater discharge rate than the late neurons. Finally, the correlation of firing activity with HR decrease in early and late neurons shows that both neurons may differently contribute to HR regulation (31).

\section{Nitric Oxide (NO) in NTS and Spontaneously Hypertension}

NO is implicated as a core regulator of central nervous circuits controlling the cardiovascular activity.
In the NTS, endothelial NO synthase (eNOS) involves in the control of the spontaneous hypertension (32).

NTS is a vital brain stem structure that has a critical role in sustaining the AP. Similarly, since NTS is the terminal area of baroreceptor afferents, it is one of the most effective central places for the modification of the baroreceptor reflex, a process that is important in blood pressure control.

Our previous study revealed that microinjection of Lglutamate into the NTS decreased blood pressure in diabetic rats, and that microinjection of lidocaine increased blood pressure (33).

Intra-NTS injection of sodium nitroprusside elevated blood pressure in diabetic rats. Subsequent to sodium nitroprusside injection, the depressing responses to glutamate declined expressively. These results showed that glucose regulates blood pressure in diabetic animals through the activation of nitric oxidergic neurons (3335). Aneffective neuromodulator of central cardiovascular autonomic activity is angiotensin II (Ang II). Within the NTS, Ang II inhibits the baroreceptor-HR reflex through the activation of type 1 receptor (12). Microinjections of Ang II into NTS make a concentration dependent effect, depressor, or pressor response. Moreover, an Ang II antagonist augmented cardiac baroreceptor reflex (32). Ang II decreases cardiac baroreceptor reflex in the NTS through Ang II type 1 receptors, which is mediated by NO. Ang II can release NO in the NTS and thus, via a phospholipase C pathway, releases intracellular calcium that is necessary for endothelial NO synthase activation (32). Released NO raises inhibitory GABAergic discharge in the NTS, which may lead to the diminution of the cardiac baroreceptor reflex. In conscious normotensive Wistar rats, long-term inhibition of eNOS in the NTS increased the cardiac baroreceptor reflex, maintaining a depressant effect of NO on the baroreceptor reflex. According to this evidence, it is supposed that NO in the NTS can be involved in the depressed baroreceptor reflex and hypertension that are produced in the spontaneously hypertensive rat (32).

NO is a diffusible molecule that can act as a central controller of food consumption, mediated by the orexin-A-induced feeding (36). NO may affect orexin neuron activity through a non-synaptic mechanism (37). Previous studies showed that microinjection of orexin into the rat NTS augmented blood pressure. Orexins produce the excitability of cardiovascular neurons in the NTS and therefore regulate global cardiovascular function (38). Studies demonstrated the presence of orexin-immunoreactive axons and the 
mRNA of both types of orexin receptors within the NTS. Orexin is a neuropeptide produced in the lateral part of the hypothalamus area and activates postsynaptic neurons via two G-protein coupled receptors $(39,40)$. Orexin is implicated in the central modification of sleep, energy metabolism, and the cardiovascular system. Central cardiovascular regulation through orexin may derive from direct actions on NTS neurons and impact neuronal excitability as a consequence of instantaneous regulation of numerous ion channels (41). In another study, it was shown that the excitatory effects of orexin on NTS neurons were mediated by activation of the phospholipase $\mathrm{C}$ and protein kinase $\mathrm{C}$ pathways, which possibly caused by orexin receptor-coupled activation of Gq (42).

During exercise, neuronal signals from the hypothalamus and the muscle receptors reach the NTS and RVLM. The signals to the RVLM produce pressor and tachycardiac responses through the activation of sympathetic premotor neurons. Without this baroreceptor reflex resetting to a higher pressure, sympathoexcitation would be decreased and parasympathetic nerves would be increased by baroreceptor inputs, causing the reduction of constant augmentations in AP and HR (43).

The NTS GABAergic interneurons may contribute to baroreceptor reflex resetting by inhibiting the barosensitive NTS neurons, and thus increase the sympathetic nerve activity. The hypothalamic paraventricular nucleus, dorsomedial hypothalamus, and tuberomammillary nucleus of the posterior hypothalamus may implicate in the inhibitory action of GABA on NTS actions (44). One of the main mechanisms for cardiovascular regulation during exercise is an increase in sympathetic nerve activity. Evidence shows that this sympathoexcitation is accomplished by the NTS as a key site in the brain (1). The core cardiovascular reflex through the NTS is the arterial baroreceptor reflex.

Baroreceptors as mechanoreceptors are excited by extending the arterial wall during AP increase. Therefore, baroreceptor afferent signals are delivered to the NTS (1). Second-order NTS glutamatergic neurons stimulate parasympathetic preganglionic cell bodies located in the nucleus ambiguus and GABAergic inhibitory neurons in the CVLM that inhibits glutamatergic neurons of RVLM, thus lessening the sympathetic preganglionic neuronal outflow. Therefore, raised parasympathetic and diminished sympathetic outflows produce a bradycardic response, decreasing the cardiac output and diminishing total peripheral resistance. These responses involve in normalizing raised AP. Opposite autonomic results, namely, diminished parasympathetic and enhanced sympathetic drive, happen when AP declines. The arterial baroreceptor reflex function shows dynamic features in response to mental and physical stress $(\mathbf{1 7 , 4 3 )}$. The NTS receives inputs from medullary raphe that regulates skin blood flow through sympathetic activation $(45,46)$. Furthermore, the NTS collects many inputs from other brain sites including periaqueductal gray area, amygdaloid complex, periaqueductal gray, hypothalamic paraventricular nucleus, and dorsomedial hypothalamus $(\mathrm{DMH})$, which regulate the NTS barosensitive neurons. Therefore, the NTS is an essential site that integrates various information sources and, as a result, affecting baroreceptor reflex functions. The integrative function of the NTS is essential for the adjustment of the baroreceptor reflex, and has a central role in regulating cardiovascular systems in mental and physical stress. Therefore, damage to the NTS is lethal because it produces cardiovascular disorder as increased variation of AP at a high level (1).

\section{Conclusion}

The NTS region of the brain stem compares the signals of cardiovascular receptors and central neural structures and normalizes the circulatory variables. Excitatory neurotransmitter, such as L-glutamate and GABAergic interneurons, are the core neurotransmitter in the regulation of AP by the NTS. The ratio of excitatory to inhibitory activity and hence sympathetic nervous action may regulate the spread of signals over the NTS neuronal network.

\section{Acknowledgments}

The authors thank Cognitive Sciences and Technologies council and Iran University of Medical Sciences for their support.

\section{Conflict of Interest}

The authors declared no potential conflicts of interest with respect to the research, authorship, and/or publication of this article.

\section{References}

1. Waki H. Central mechanisms of cardiovascular regulation during exercise: Integrative functions of the nucleus of the solitary tract. J Physical Fitness Sports Med. 2012;1(2):253-61. [DOI:10.7600/jpfsm.1.253]

2. Zanutto BS, Valentinuzzi ME, Segura ET. Neural set point for the control of arterial pressure: role of the nucleus tractus solitarius. BiomedEngineer Online. 2010;9(1):4. [DOI:10.1186/1475-925X-9-4] 
3. Paton J, Spyer K. Central nervous control of the cardiovascular system. Autonomic Failure: A Textbook of Clinical Disorders of the Autonomic Nervous System. 2013:35-51. [DOI:10.1093/med/9780198566342.003.0004]

4. O'hagan KP, Casey SM, Clifford PS. Muscle chemoreflex increases renal sympathetic nerve activity during exercise. J Apply Physiol. 1997;82(6):1818-25. [DOI:10.1152/jappl.1997.82.6.1818]

5. Osborn JW, Jacob F, Guzman P. A neural set point for the long-term control of arterial pressure: beyond the arterial baroreceptor reflex. Am J PhysioRegInteg Compar Physiol. 2005;288(4):R846-R55. [DOI:10.1152/ajpregu.00474.2004]

6. Kourosh Arami M, Sarihi A, Heshmatian B, Malakouti $\mathrm{S}$, Amiri I. The effect of nucleus tractus solitarius inactivation on blood pressure in diabetic rats. Iran JPharmaceut Res. 2004:(3)1:76.

7. Cutsforth-Gregory JK, Benarroch EE. Nucleus of the solitary tract, medullary reflexes, and clinical implications. Neurol. 2017;88(12):1187-96. [DOI:10.1212/WNL.0000000000003751]

8. Kawai Y. Differential ascending projections from the male rat caudal nucleus of the tractus solitarius: an interface between local microcircuits and global macrocircuits. FrontNeuroanat. 2018;12:63. [DOI:10.3389/fnana.2018.00063]

9. Cui S, Wang K, Wu SB, et al. Electroacupuncture modulates the activity of the hippocampus-nucleus tractus solitarius-vagus nerve pathway to reduce myocardial ischemic injury. Neural Regenerat Res. 2018;13(9):1609. [DOI:10.4103/1673-5374.237124]

10. Lee HY, Oh KH, Yang EK, Ahn DK, Lee WJ, Park JS. Cardiovascular effects of endogenous GABA in the nucleus tractus solitarius. Korean Circ J. 1997;27(1):94101. [DOI:10.4070/kcj.1997.27.1.94]

11. Zubcevic J, Potts JT. Role of GABAergic neurones in the nucleus tractus solitarii in modulation of cardiovascular activity. Experiment Physiol. 2010;95(9):909-18.

[DOI:10.1113/expphysiol.2010.054007]

12. Carter DA, Choong YT, Connelly AA, et al. Functional and neurochemical characterization of angiotensin type 1A receptor-expressing neurons in the nucleus of the solitary tract of the mouse. Am J PhysiolRegul Integ Comp Physiol. 2017;313(4):R438-R49. [DOI:10.1152/ajpregu.00168.2017]

13. Machado BH, Mauad H, Chianca Jr D, Haibara A, Colombari E. Autonomic processing of the cardiovascular reflexes in the nucleus tractus solitari. Braz J Med Biol Res. 1997;30:533-5. [DOI:10.1590/S0100-879X1997000400015]
14. Komaki A, Shahidi S, Sarihi A, et al. Effects of neonatal C-fiber depletion on interaction between neocortical short-term and long-term plasticity. Basic Clin Neurosci. 2013;4(2):136-145

15. Arami MK, Hajizadeh S, Semnanian S. Postnatal development changes in excitatory synaptic activity in the rat locus coeruleus neurons. Brain Res. 2016;1648:365-71.

[DOI:10.1016/j.brainres.2016.07.036]

16. Arami MK, Semnanian S, Javan M, Hajizadeh S, Sarihi A. Postnatal developmental alterations in the locus coeruleus neuronal fast excitatory postsynaptic currents mediated by ionotropic glutamate receptors of rat. Physiol Pharmacol. 2011;14(4):337-48.

17. Sapru HN. Neurotransmitters in the nucleus tractus solitarius mediating cardiovascular function. Neural mechanisms of cardiovascular regulation: Springer; 2004. p. 81-98. [DOI:10.1007/978-1-4419-9054-9 4]

18. Erickson JD, De Gois S, Varoqui H, Schafer MK-H, Weihe E. Activity-dependent regulation of vesicular glutamate and GABA transporters: a means to scale quantal size. NeurochemInt. 2006;48(6-7):643-9. [DOI:10.1016/j.neuint.2005.12.029]

19. Kilman V, Van Rossum MC, Turrigiano GG. Activity deprivation reduces miniature IPSC amplitude by decreasing the number of postsynaptic GABAA receptors clustered at neocortical synapses. J Neurosci. 2002;22(4):1328-37. [DOI:10.1523/JNEUROSCI.2204-01328.2002]

20. Spary EJ, Maqbool A, Saha S, Batten TF. Increased GABA B receptor subtype expression in the nucleus of the solitary tract of the spontaneously hypertensive rat. J Molec Neurosci. 2008;35(2):211-24. [DOI:10.1007/s12031-008-9055-9]

21. Zhang W, Herrera-Rosales M, Mifflin S. Chronic hypertension enhances the postsynaptic effect of baclofen in the nucleus tractus solitarius. Hypertension. 2007;49(3):659-63.

[DOI:10.1161/01.HYP.0000253091.82501.c0]

22. Potts J, Paton J, Mitchell J, et al. Contraction-sensitive skeletal muscle afferents inhibit arterial baroreceptor signalling in the nucleus of the solitary tract: role of intrinsic GABA interneurons. Neurosci. 2003;119(1):201-14. [DOI:10.1016/S03064522(02)00953-3]

23. Mifflin SW. What does the brain know about blood pressure? Physiology. 2001;16(6):266-71. [DOI:10.1152/physiologyonline.2001.16.6.266]

24. Derera ID, Delisle BP, Smith BN. Functional neuroplasticity in the nucleus tractus solitarius and increased risk of sudden death in mice with acquired temporal lobe epilepsy. eNeuro. 2017;4(5). [DOI:10.1523/ENEURO.0319-17.2017] 
25. Derera ID, Smith KC, Smith BN. Altered A-type potassium channel function in the nucleus tractus solitarii in acquired temporal lobe epilepsy. JNeurophysiol. 2019;121(1):177-87. [DOI:10.1152/jn.00556.2018]

26. Kolpakova J, Li L, Hatcher JT, et al. Responses of nucleus tractus solitarius (NTS) early and late neurons to blood pressure changes in anesthetized F344 rats. PloS one. 2017;12(4). [DOI:10.1371/journal.pone.0169529]

27. Rogers RF, Paton J, Schwaber JS. NTS neuronal responses to arterial pressure and pressure changes in the rat. AmJ PhysiolReg IntegCompar Physiol. 1993;265(6):R1355-R68. [DOI:10.1152/ajpregu.1993.265.6.R1355]

28. Zhang J, Mifflin SW. Responses of aortic depressor nerve-evoked neurones in rat nucleus of the solitary tract to changes in blood pressure. JPhysiol. 2000;529(2):431-43. [DOI:10.1111/j.14697793.2000.00431.x ]

29. Deuchars J, Li YW, Kasparov S, Paton JF. Morphological and electrophysiological properties of neurones in the dorsal vagal complex of the rat activated by arterial baroreceptors. J Compar Neurol. 2000;417(2):233-49.

https://doi.org/10.1002/(SICI)1096-

9861(20000207)417:2<233::AID-CNE8>3.0.CO;2-V

[DOI:10.1002/(SICI)1096-

9861(20000207)417:23.0.CO;2-V]

30. Dampney RA, Polson JW, Potts PD, Hirooka Y, Horiuchi J. Functional organization of brain pathways subserving the baroreceptor reflex: studies in conscious animals using immediate early gene expression. Cell Mol Neurobiol. 2003; 23:597-616. [DOI:10.1023/A:1025080314925]

31. Gu H, Lin M, Liu J, et al. Selective impairment of central mediation of baroreflex in anesthetized young adult Fischer 344 rats after chronic intermittent hypoxia. Am J PhysiolHeart Circ Physiol. 2007;293(5):H2809H18. [DOI:10.1152/ajpheart.00358.2007]

32. Waki H, Murphy D, Yao ST, Kasparov S, Paton JF. Endothelial NO synthase activity in nucleus tractus solitarii contributes to hypertension in spontaneously hypertensive rats. Hypertension. 2006;48(4):644-50. [DOI:10.1161/01.HYP.0000238200.46085.c6]

33. Kourosh Arami M, Sarihi A, Malacoti SM, Behzadi G, Vahabian M, Amiri I. The effect of nucleus tractus solitarius nitric oxidergic neurons on blood pressure in diabetic rats. Iran BiomedJ.2006;10(1):15-9.

34. Arami MK, Sarihi A, Behzadi J, Malakouti SM, Amiri I, Ekbatani RZ. The effect of hyperglycemia on nitric oxidergic neurons in nucleus tractus solitarius and blood pressure regulation in rats with induced diabetes. Iran J DiabetLipid Disorder. 2005;4(3):E2.
35. Dias ACR, Vitela M, Colombari E, Mifflin SW. Nitric oxide modulation of glutamatergic, baroreflex, and cardiopulmonary transmission in the nucleus of the solitary tract. Am J PhysiolHeart Circ Physio. 2005;288(1):H256-H62. [DOI:10.1152/ajpheart.01149.2003]

36. Farr SA, Banks WA, Kumar VB, Morley JE. OrexinA-induced feeding is dependent on nitric oxide. Peptides. [DOI:10.1016/j.peptides.2004.12.004]

37. Cutler DJ, Morris R, Evans ML, Leslie RA, Arch JR, Williams G. Orexin-A immunoreactive neurons in the rat hypothalamus do not contain neuronal nitric oxide synthase (nNOS). Peptides. 2001;22(1):123-8. [DOI:10.1016/S0196-9781(00)00364-8]

38. Smith PM, Connolly BC, Ferguson AV. Microinjection of orexin into the rat nucleus tractus solitarius causes increases in blood pressure. Brain Res. 2002;950(12):261-7. [DOI:10.1016/S0006-8993(02)03048-2]

39. Babasafari M, Kourosharami M, Behman J, Farhadi M, Komaki A. Alteration of phospholipase C expression in rat visual cortical neurons by chronic blockade of orexin receptor 1. Int J Pept Res Ther.2019:1-7. [DOI:10.1007/s10989-019-09943-y]

40. Rezaei Z, Kourosh-Arami M, Azizi H, Semnanian S. Orexin type-1 receptor inhibition in the rat lateral paragigantocellularis nucleus attenuates development of morphine dependence. Neurosci Lett. 2020:724:134875.

[DOI:10.1016/j.neulet.2020.134875]

41. Yang B, Ferguson AV. Orexin-A depolarizes nucleus tractus solitarius neurons through effects on nonselective cationic and $\mathrm{K}+$ conductances. JNeurophysiol. 2003;89(4):2167-75. [DOI:10.1152/jn.01088.2002]

42. Yang B, Samson WK, Ferguson AV. Excitatory effects of orexin-A on nucleus tractus solitarius neurons are mediated by phospholipase $\mathrm{C}$ and protein kinase $\mathrm{C}$. $\mathrm{J}$ Neurosci. 2003;23(15):6215-22. [DOI:10.1523/JNEUROSCI.23-15-06215.2003]

43. Waki H, Takagishi M, Gouraud SS. Central mechanisms underlying anti-hypertensive effects of exercise training. J Physic Fit Sports Med. 2014;3(3):317-25. [DOI:10.7600/jpfsm.3.317]

44. Potts JT. Inhibitory neurotransmission in the nucleus tractus solitarii: implications for baroreflex resetting during exercise. Experiment Physiol. 2006;91(1):59-72. [DOI:10.1113/expphysiol.2005.032227]

45. Arami MK. Nitric oxide in the nucleus raphe magnus modulates cutaneous blood flow in rats during hypothermia. Iran J Basic MedSci. 2015;18(10):989. 
46. Malakouti SM, Kourosh AM, Sarihi A, et al. Reversible inactivation and excitation of nucleus raphe magnus can modulate tail blood flow of male wistar rats in response to hypothermia. Iran Biomed J.2008;12(4):237-40

\section{How to Cite This Article:}

Kourosharami M, Mohsenzadegan M, Komaki A. A Review of Excitation-Inhibition Balance in the Nucleus Tractus Solitarius as a Gateway to Neural Cardiovascular Regulation. J Adv Med Biomed Res. $2020 ; 28$ (126) $: 47-53$

\section{Download citation:}

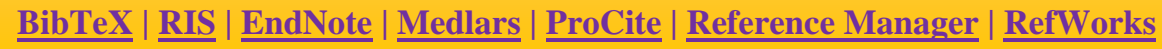

\section{Send citation to:}

9. Mendeley 2 Zotero RefWorks RefWorks 